\title{
Teasaponin suppresses Candida albicans filamentation by reducing the level of intracellular cAMP
}

\author{
Ying $\mathrm{Li}^{1}$, Mingzhu Shan ${ }^{1}$, Shihui $\mathrm{Li}^{1}$, Yuechen Wang ${ }^{2}$, Huan Yang ${ }^{1}$, Ying Chen ${ }^{1}$, Bing Gu ${ }^{1,3}$, Zuobin Zhu ${ }^{2}$ \\ ${ }^{1}$ Medical Technology School of Xuzhou Medical University, Xuzhou Key Laboratory of Laboratory Diagnostics, Xuzhou 221004, China; \\ ${ }^{2}$ Department of Genetics, Xuzhou Medical University, Xuzhou 214200, China; ${ }^{3}$ Department of Laboratory Medicine, Affiliated Hospital of Xuzhou \\ Medical University, Xuzhou 221006, China \\ Contributions: (I) Conception and design: Y Li, Z Zhu; (II) Administrative support: B Gu; (III) Provision of study materials: H Yang, Y Chen; (IV) \\ Collection and assembly of data: M Shan, S Li, Y Wang; (V) Data analysis and interpretation: Y Li, M Shan, Z Zhu; (VI) Manuscript writing: All \\ authors; (VII) Final approval of manuscript: All authors. \\ Correspondence to: Bing Gu. Department of Laboratory Medicine, Affiliated Hospital of Xuzhou Medical University, Xuzhou 221006, China. \\ Email: gb20031129@163.com; Zuobin Zhu. Department of Genetics, Xuzhou Medical University, Xuzhou 214200, China. Email: geneticszzb@163.com.
}

Background: Candidiasis has long been a threat to human health, but cytotoxicity and resistance always block the usefulness of antifungal agents. The ability to switch between yeast and hypha is one of the most discussed virulence trait attributes of the human pathogenic fungus Candida albicans. The morphological transition provides a novel target for developing antifungal drugs. The aim of the present study was to explore the activity and mechanism of teasaponin (TS), a generally regarded as safe natural product, in inhibiting filamentation of C. albicans, hoping to provide an experimental basis for its clinical application.

Methods: The effect of TS on filamentation and biofilm formation of $C$. albicans was evaluated by XTT reduction assay and microscopy. The level of intracellular cAMP was measured to further explore the underlying mechanism. In addition, cytotoxicity of TS was evaluated by using MTT assay in vitro and Caenorhabditis elegans model in vivo. The potential of TS-resistance induction was tested by a serial passage experiment.

Results: TS displayed a moderate antifungal activity against the wild type, efflux pump mutant and multiresistance $C$. albicans strains, and could effectively retard filamentation and biofilm formation with a low MIC value. Further mechanism investigation revealed that the reduced cAMP level inhibited filamentation and biofilm formation. In addition, TS showed no significant cytotoxicity in vitro or in vivo, and had little potential to develop resistance during long-time induction.

Conclusions: Our work evaluated the antifungal activity of TS against filamentation and biofilms formation of C. albicans and disclosed the underlying mechanism, which might provide useful clues for the potential clinical application of TS in fighting clinical fungal infections by targeting the virulence factors.

Keywords: Teasaponin; Candida albicans; filamentation; cAMP

Submitted Jul 25, 2019. Accepted for publication Jan 02, 2020.

doi: 10.21037/atm.2020.01.124

View this article at: http://dx.doi.org/10.21037/atm.2020.01.124

\section{Introduction}

Some risk factors, including the use of immunosuppressive, cytotoxic drugs, powerful antibiotics and various implanted devices, make opportunistic fungal pathogens liable to attack immunocompromised or some other debilitated hosts (1). Candida species are the fourth most common pathogens causing hospital-acquired systemic infections in the United States, with crude mortality rates as high as $50 \%$ (2). Meanwhile, it is estimated that up to $75 \%$ of women experienced at least one episode of vulvovaginal candidiasis (VVC) in their lifetime (3), of whom, 5-8\% experienced 
Table 1 The MIC of TS and FLC against different genotypes of $C$. albicans in vitro

\begin{tabular}{llc}
\hline \multirow{2}{*}{ Drugs and strains $^{\alpha}$} & \multicolumn{2}{c}{ MICs of drugs $(\mu \mathrm{g} / \mathrm{mL})^{\beta}$} \\
\cline { 2 - 3 } & TS & FLC \\
\hline ATCC10231 & 64 & 1 \\
SC5314 & 64 & 2 \\
YEM30 & 64 & 2 \\
DSY448 & 64 & 0.5 \\
DSY1050 & 64 & 0.5 \\
CA10 & 64 & $>128$ \\
CA148 & 64 & $>128$ \\
CCF3 & 64 & 1 \\
HLY3426 & 64 & 1 \\
\hline
\end{tabular}

${ }^{\alpha}$, ATCC10231, SC5314 and YEM30 are wild-type strains. The genotype of DSY448 is $\Delta c d r 1:: h i s G-U R A 3-h i s G / \Delta c d r 1:: h i s G ;$ The genotype of DSY1050 is $\Delta c d r 1:$ hisG/ $\Delta c d r 1:: h i s G \Delta c d r 2:$ hisG/ $\Delta c d r 2:$ hisG $\triangle m d r 1:: h i s G-U R A 3-h i s G / \Delta m d r 1:$ hisG (14). CA10 and CA148 are clinical isolated azoles multi-resistance $C$. albicans isolates (15). The genotype of CCF3 is ura3::1 imm434/ura3::1 imm434 flo8::hisG/flo8::hisG-URA3-hisG; The genotype of HLY3426 is ura3::1 imm434/ura3::1 imm434 flo8::hisG/flo8::hisG RP10::ACT1p-FLO8-13MYCFLAG-URA3 (16). ${ }^{\beta}$, TS, teasaponin; FLC, fluconazole.

at least four episodes of chronic recurrences per year (4). Candida albicans is the most common human fungal pathogen, causing both superficial and invasive candidiasis (5). Usually, C. albicans is a benign member of the human gastrointestinal and genital tracts. However, if the host immune function is impaired or the environmental niche is available, C. albicans can cause debilitating mucosal or fatal systemic infections via exerting its own virulence factors (6).

The dimorphic phenotype, ranging from budding yeast to hyphae, is a striking feature of $C$. albicans. Yeast cells, as a disseminating form, play a critical role in the early stage of the infection process for adhesion on the tissue surface. Hyphal cells, as an important pathogenic form, have the advantage of escaping from the host immune system and invading the tissue in the pathogenesis (7). This morphological plasticity is a crucial perpetrator of candidiasis. In addition, structured biofilm consists of yeast, hyphae and self-produced extracellular matrix with high drug-resistance up to 1,000 folds (8). C. albicans is equipped with a variety of virulence traits and escape strategies, helping them colonize within the commensal microbiota and invade the host tissue during infection. The expression of adhesins and invasins, the plasticity of morphology, the formation of biofilm, contact sensing, phenotypic switching and the secretion of hydrolytic enzymes are some important virulence properties (9). Thus, antivirulence, especially the formation of hyphae and biofilm, is a powerful therapeutic strategy for the management of C. albicans infection (10).

Like antibiotics, antifungal drugs also face the problem of drug resistance and toxicity that block the treatment of fungal infection. Natural products have long been recognized as an excellent source of new drugs. Statistically, from 1981 to 2014, nearly $60 \%$ of small-molecule drugs approved by the U.S. Food and Drug Administration (FDA) were either natural products or based thereon (11). Teasaponin (TS), generally regarded as a safe compound, is a tea seed-derived natural surfactant with various bioactivities. Previous studies $(12,13)$ have demonstrated that TS possesses many pharmacological functions and fine foaming and emulsifying activity for pesticides. In this study, we found that TS could effectively inhibit the formation of hyphae and biofilm of C. albicans. Further mechanism research showed that the reduction of cyclic adenosine monophosphate (cAMP) level might contribute to the inhibitory effect of TS.

\section{Methods}

\section{Strains, culture and chemicals}

C. albicans strains used in this study are shown in Table 1 (14-16). C. albicans strain ATCC10231 was obtained from the ATCC Essentials of Life Science Research, USA. Wild type Caenorbabditis elegans strain N2 was obtained from the Caenorhabditis Genetics Center, USA. Clinical derived C. albicans azole-resistant isolates CA10 and CA148 were donated by Shandong Provincial Qianfoshan Hospital (15). All strains were stocked and prepared as our lab previously described (17). Fluconazole (FLC) and dibutyryl-cAMP (db-cAMP) were purchased from Sigma (St Louis, USA), fetal bovine serum (FBS) was purchased from Gibco (USA), and TS was purchased from Aladdin (Shanghai, China). They were dissolved in dimethyl sulfoxide (DMSO) (Sigma, St Louis, USA) at 10 or $50 \mathrm{mg} / \mathrm{mL}$. In each assay, the content of DMSO was below $0.5 \%$.

\section{Minimal inbibitory concentration (MIC) test}

The MIC value of TS against different genotypes of $C$. albicans was determined by the broth microdilution method 
according to the Clinical and Laboratory Standards Institute (CLSI) guidelines (M27-A3) (18). The minimal concentration of zero visible growth was considered as the endpoint value.

\section{Inbibitory curve of proliferation}

Wild type strain YEM30 was diluted in synthetic medium, plus dextrose (SD medium) with an initial inoculum of $1 \times 10^{5}$ cells $/ \mathrm{mL}$. TS was added to the final concentrations of $0,16,32$, and $64 \mu \mathrm{g} / \mathrm{mL}$, and $2 \mu \mathrm{g} / \mathrm{mL}$ FLC served as positive control group. Cells were cultured at $30{ }^{\circ} \mathrm{C}$ with shaking at $200 \mathrm{rpm}$ for 48 hours. Every 2 hours, $100-\mu \mathrm{L}$ aliquots of samples were transferred to a 96-well flat-bottom plate and detected for absorbance at $600 \mathrm{~nm}$ with a Bio-Rad microplate reader (Bio-Rad Laboratories, Richmond, CA).

\section{Inbibition of filamentation}

The effect of TS on the filamentation of wild type strain YEM30 in different culture media was detected using a modified broth microdilution method as previously described (19). Cells containing various doses of TS (0, 16,32 and $64 \mu \mathrm{g} / \mathrm{mL}$ ) were diluted in RPMI1640 medium (pH7.0, buffered with MOPS), RPMI1640 plus 5\% FBS medium, spider medium (1\% nutrient broth, $0.5 \%$ mannitol, $0.1 \% \mathrm{~K}_{2} \mathrm{HPO}_{4}$, $\mathrm{pH} 7.2$ ) and spider plus 5\% FBS medium, respectively, at $1 \times 10^{5}$ cells $/ \mathrm{mL}$ and incubated at $37{ }^{\circ} \mathrm{C}$ without shaking. Hyphal development was monitored by a microscope (Olympus IX71, Olympus, Tokyo, Japan) in the bright field mode at specific times (3, 6, 9 and $12 \mathrm{~h}$ ).

\section{Inbibition of biofilm formation}

The effect of TS against biofilm formation was measured using an XTT reduction assay and microscopic observation as previously described (20). $100 \mu \mathrm{L}$ YEM30 (wild type strain), CCF3 (flo8/flo 8 mutant) and HLY3426 (flo $8 / f l o 8$ + FLO8 mutant) (16) cell suspensions $\left(1 \times 10^{6}\right.$ cells $/ \mathrm{mL}$ in RPMI1640 medium or RPMI1640 plus 5\% FBS medium) containing various doses of TS $(0,32,64$ and $128 \mu \mathrm{g} / \mathrm{mL})$ were seeded into a 96 -well flat-bottom plate. The plate was incubated at $37^{\circ} \mathrm{C}$ for $24 \mathrm{~h}$ in static culture. After three washes with sterile PBS (0.01 M, pH 7.2), biofilm formation was measured with an XTT Cell Proliferation Assay Kit (BestBio, Shanghai, China) according to the provided protocol and observed under an Olympus microscope in the bright field mode.

\section{Determination of intracellular cAMP content}

The effect of TS on the intracellular cAMP concentrations was measured as previously described with some modifications (21). YEM30 cells $\left(1 \times 10^{6}\right.$ cells $\left./ \mathrm{mL}\right)$ with various doses of TS were cultured in RPMI1640 medium at $37^{\circ} \mathrm{C}$ for $12 \mathrm{~h}$, and $2 \mu \mathrm{g} / \mathrm{mL}$ FLC served as positive control group. Cells were harvested by centrifugation, washed 3 times with deionized water, weighed the dry weight, frozen in liquid nitrogen and thawed at room temperature repeatedly, and finally suspended in deionized water with 5\% trichloroacetic acid. Following breaking by ultrasonication, the supernatant was neutralized with water-saturated ether five times and then subjected to freeze-drying. The content of intracellular cAMP was measured using a cAMP ELISA Kit (Elabscience Biotechnology Co., Ltd, Guangdong, China) according to the provided protocol. The concentrations of cAMP were modified by their cell weights.

\section{cAMP rescue test}

To test the effect of cAMP on hyphal formation after TS treatment, YEM30 cells were prepared as the above filamentation experiment. db-cAMP was added into $32 \mu \mathrm{g} / \mathrm{mL}$ TS and drug-free treatment group at a final concentration of $1 \mathrm{mM}$. The db-cAMP free group served as control. After 6 -h incubation at $37^{\circ} \mathrm{C}$, the morphology of formed hyphae was observed under an Olympus microscope.

The effect of cAMP on biofilm formation in the presence of TS was tested as previously described (20) with some modifications. YEM30 cells were prepared as the above biofilm formation experiment. db-cAMP was added to $64 \mu \mathrm{g} / \mathrm{mL}$ and drug-free treatment groups at a final concentration of $1 \mathrm{mM}$. The $\mathrm{db}$-cAMP free group served as control. After $24-\mathrm{h}$ incubation at $37^{\circ} \mathrm{C}$, the formed biofilm was detected by XTT reduction assay and microscopic observation.

\section{Transcriptional levels of hypha-specific genes}

Quantitative real-time RT-PCR was used to investigate the transcriptional levels of hypha-specific genes under TS treatment. As previously described (20), YEM30 
Table 2 Gene-specific primers used for real-time RT-PCR

\begin{tabular}{ll}
\hline Primers & \multicolumn{1}{c}{ Sequence } \\
\hline RAS1-F & GGCCATGAGAGAACAATATA \\
RAS1-R & GTCTTTCCATTTCTAAATCAC \\
ALS3-F & CTAATGCTGCTACGTATAATT \\
ALS3-R & CCTGAAATTGACATGTAGCA \\
HWP1-F & TGGTGCTATTACTATTCCGG \\
HWP1-R & CAATAATAGCAGCACCGAAG \\
CDC35-F & TTCATCAGGGGTTATTTCAC \\
CDC35-R & CTCTATCAACCCGCCATTC \\
PDE2-F & ACCACCACCACTACTACTAC \\
PDE2-R & AAAATGAGTTGTTCCTGTCC \\
EFG1-F & TATGCCCCAGCAAACAACTG \\
EFG1-R & TTGTTGTCCTGCTGTCTGTC \\
ECE1-F & GCTGGTATCATTGCTGATAT \\
ECE1-R & TTCGATGGATTGTTGACAC \\
$18 S ~ r R N A-F$ & AATTACCCAATCCCGACAC \\
$18 S ~ r R N A-R$ &
\end{tabular}

cells were diluted in RPMI1640 medium with or without $32 \mu \mathrm{g} / \mathrm{mL}$ of TS. After an incubation of 6 -h at $37^{\circ} \mathrm{C}$, cells were harvested and washed with sterile water. The hot phenol method was used to extract the total RNAs, and the cDNA was synthesized by a cDNA synthesis kit (TaKaRa Biotechnology, Dalian, China). PCR reactions were performed using a SYBR Green master mix (TaKaRa Biotechnology, Dalian, China) in a Roche Real-Time PCR System. Primer sequences are listed in Table 2. The $18 \mathrm{~S}$ $r R N A$ served as the internal control gene.

\section{Induction of TS resistance}

The capacity of TS resistance induction was tested as previously described with some modifications (19). Briefly, YEM30 cells were inoculated in YPD broth and cultured at $30{ }^{\circ} \mathrm{C}, 200 \mathrm{rpm}$ with $32 \mu \mathrm{g} / \mathrm{mL}$ TS, a concentration half of MIC inhibiting hyphal formation. Every day, $100 \mu \mathrm{L}$ of each culture was serially transferred into $10 \mathrm{~mL}$ YPD broth (yeast extract $1 \%$, peptone $2 \%$ and dextrose $2 \%$ ) containing $32 \mu \mathrm{g} / \mathrm{mL}$ TS, and the everyday transfer was performed for 40 days. Each day, cultures were withdrawn to test their MICs.

\section{Toxicity assay}

Two normal cell lines and wild type C. elegans were used to evaluate the cytotoxicity of TS.

The effect of TS against the proliferation of cell line HT22 (mouse hippocampal cell line) and HEK 293T (human embryonic kidney cell line) was detected by MTT assay as previously described (22). After 24-h incubation, the treated cells were incubated with MTT $(0.5 \mathrm{mg} / \mathrm{mL})$ for additional $4 \mathrm{~h}$ in the dark. The spectrophotometric absorbance of each well was measured at $570 \mathrm{~nm}$ using a Bio-Rad plate reader. The $\mathrm{IC}_{50}$ value was calculated based on the percentage of viable cells.

The effect of TS on the survival of wild type $C$. elegans N2 was also tested as previously described (20). Briefly, different concentrations of TS were added to the prepared nematodes in a 96-well plate. After 2-day incubation at $25^{\circ} \mathrm{C}$, the treated worms were monitored by microscopic observation, and the number of living and dead worms were recorded to calculate the survival rate.

\section{Statistical analysis}

All experiments were assayed in triplicate on a given day and repeated on additional two days. All results are represented as the mean values with the corresponding standard deviations (SD) of triplicate measurements from three independent experiments. Statistical significance was analyzed by Student's $t$-test (two-tailed, unequal variance). $\mathrm{P}<0.05$ was considered as statistically significant.

\section{Results}

\section{TS inhibits the proliferation of C. albicans}

To evaluate the effect of TS against the proliferation of $C$. albicans, the MIC value of TS against different genotypes of C. albicans was assayed in vitro. As shown in Table 1, TS displayed moderate antifungal activity against $C$. albicans, and the MIC values of all tested strains was $64 \mu \mathrm{g} / \mathrm{mL}$, whether it be wild type strain (ATCC10231, SC5314 and YEM30) or efflux pump mutant strain (DSY448 and DSY1050) (14) or multi-resistance strain (CA10 and CA148) (15) or avirulent strain (CCF3, flo $8 /$ flo 8 mutant) and its complementation strain (HLY3426, flo $/$ flo $8+$ FLO 8 mutant) (16). The MIC of FLC (positive control) was $0.5-$ $>128 \mu \mathrm{g} / \mathrm{mL}$ for different strains.

In addition, the proliferation inhibitory curve of TS was 


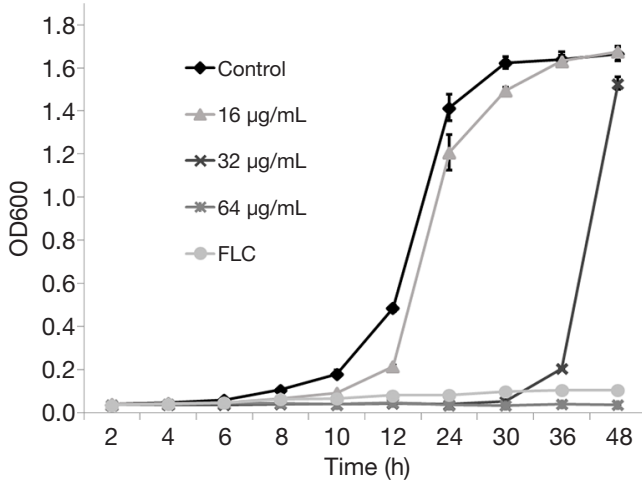

Figure 1 The growth curve of wild type C. albicans strain YEM30 under TS treatment. YEM30 cells were diluted in the SD medium and incubated with different doses of TS at $30{ }^{\circ} \mathrm{C}$ with shaking. OD600 of each group was detected at a 2-h interval using a BioRad microplate reader. The results are shown as means \pm standard deviation (SD).

also plotted over $48 \mathrm{~h}$. The results showed that $16 \mu \mathrm{g} / \mathrm{mL}$ TS (1/4 MIC) could retard the growth of C. albicans as compared with drug-free control group over $24 \mathrm{~h}$. The growth curve of 32 and $64 \mu \mathrm{g} / \mathrm{mL}$ was similar to that of $2 \mu \mathrm{g} / \mathrm{mL}$ of FLC (positive control), almost completely suppressing the proliferation in $24 \mathrm{~h}$, and during the treatment with $32 \mu \mathrm{g} / \mathrm{mL}$ TS, C. albicans resumed growth after $36 \mathrm{~h}$ (Figure 1). We collected the treated cells with 32 or $64 \mu \mathrm{g} / \mathrm{mL}$ TS for $48 \mathrm{~h}$ and measured their MIC values of TS. Meanwhile, the collected cells were stained by propidium iodide (PI) to determine viable or dead cells. Results showed that the MIC values of TS had no change after TS treatment for $48 \mathrm{~h}$, implying no resistance formation. PI could stain most cells of $64 \mu \mathrm{g} / \mathrm{mL}$ TS treated cells in red fluorescence, while few cells treated by $32 \mu \mathrm{g} / \mathrm{mL}$ TS could be penetrated by PI (data not shown). These results implied that TS could inhibit the proliferation of C. albicans, the resumed growth of $32 \mu \mathrm{g} / \mathrm{mL}$ TS group might due to the degradation of TS reducing its antifungal activity, and high concentration of TS exerted fungicidal activity against $C$. albicans.

\section{TS retards the hyphal growth of C. albicans}

Knowing that hyphal growth is the most recognized virulence factor during the C. albicans infection process, we examined the effect of TS on the hyphal growth in vitro by microphotography. As illustrated in Figure $2 \mathrm{~A}$, hyphal initiation and elongation were observed in control and $16 \mu \mathrm{g} / \mathrm{mL}$ groups in RPMI 1640 medium within $9 \mathrm{~h}$, and extensive biofilm formation were observed at $12 \mathrm{~h}$. In contrast, cells in $32 \mu \mathrm{g} / \mathrm{mL}$ group grew as a yeast form within $9 \mathrm{~h}$, and the length of formed hyphae at $12 \mathrm{~h}$ was much shorter than that in control and $16 \mu \mathrm{g} / \mathrm{mL}$ groups. The yeast form remained unchanged within $12 \mathrm{~h}$ in the $64 \mu \mathrm{g} / \mathrm{mL}$ and FLC treatment (positive control) groups.

We also examined the hyphal growth in other hyphainduced cultures, RPMI 1640 plus 5\% FBS medium, spider medium and spider plus 5\% FBS medium. After 9-h culture at $37^{\circ} \mathrm{C}$, hyphal growth in the spider medium was similar as that in RPMI1640 medium. Meanwhile, most cells of $64 \mu \mathrm{g} / \mathrm{mL}$ TS group in plus FBS medium still retained yeast form just like those in the positive control FLC group. In addition, the number and length of hyphae in 32 and $64 \mu \mathrm{g} / \mathrm{mL}$ groups were much smaller and shorter than those in control group (Figure 2B). This phenomena implied that TS had the capacity to inhibit hyphal formation of $C$. albicans.

\section{TS inbibits the biofilm formation of C. albicans}

Knowing that hyphal growth plays a crucial role in biofilm formation, and high drug resistance and host immune escape are key virulence factors contributing to biofilm formation (23), we examined whether TS had an impact on the biofilm formation of wild type and avirulent strains in different medium using microphotography and XTT reduction assay. Microscopic observation revealed that the formed biofilm in wild type strain YEM30 were composed of a mixture of hyphae and yeast cells in non-treated and $32 \mu \mathrm{g} / \mathrm{mL}$ groups. In contrast, only clusters of yeast or short hyphae were observed on the bottom of the well without biofilm structures in 64 and $128 \mu \mathrm{g} / \mathrm{mL}$ groups (Figure $3 A$ ). The XTT assay result showed that the inhibitory effect was dose-dependent, as reflected by a decrease in metabolic activity. Specifically, $64 \mu \mathrm{g} / \mathrm{mL}$ TS could significantly prevent about $80 \%$ biofilm formation (Figure $3 B, \mathrm{P}<0.01$ ). Under the filamentous-induced condition (RPMI 1640 plus FBS medium), although there were more formed biofilm or cells in 64 or $128 \mu \mathrm{g} / \mathrm{mL}$ groups as compared with the control group under the filamentous-induced condition (plus FBS) (Figure 3A), 64 and $128 \mu \mathrm{g} / \mathrm{mL}$ TS could significantly inhibit biofilm formation (Figure $3 B, \mathrm{P}<0.01$ ). Meanwhile, the inhibitory tendency of non-filamentous strain CCF3 and its complementation strain HLY3426 was similar to the wild type strain. These data implied that TS had a negative effect on biofilm formation of $C$. albicans. 

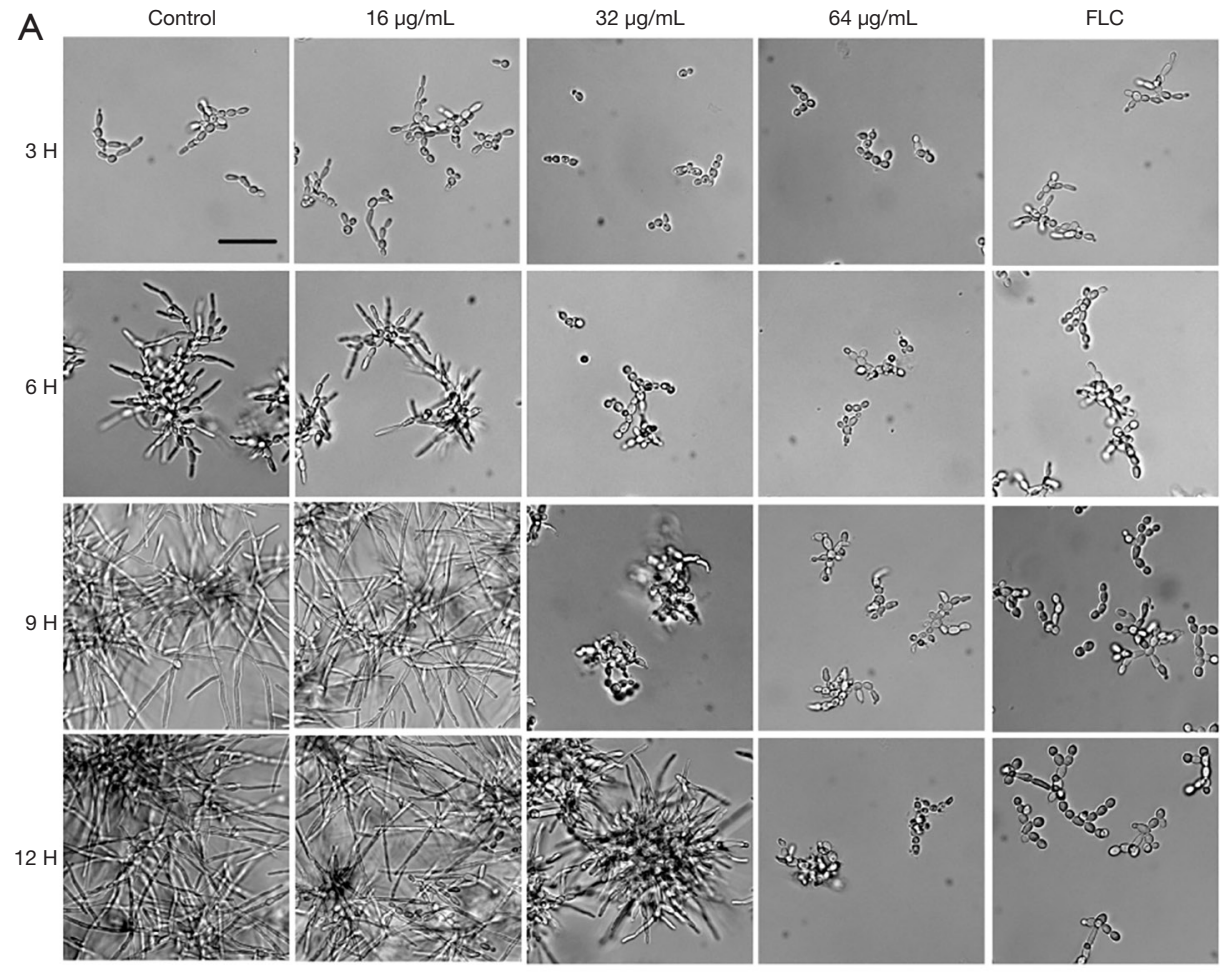

B

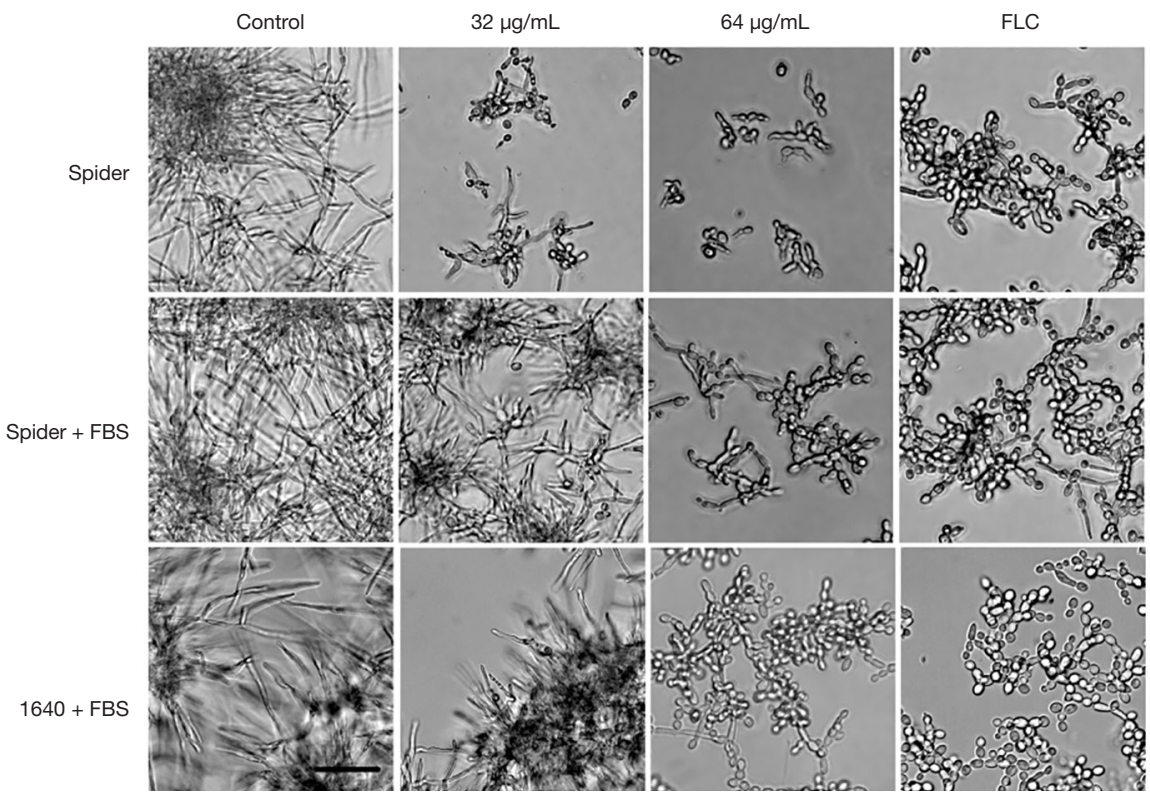

Figure 2 The effect of TS on the filamentation of C. albicans. (A) YEM30 cells were diluted in the RPMI1640 medium and incubated with different doses of TS at $37^{\circ} \mathrm{C}$ without shaking. Every three hours, cells were imaged under a microscope in the bright field. $2 \mu \mathrm{gg} / \mathrm{mL}$ FLC served as positive control. (B) YEM30 cells were diluted in spider medium, spider plus 5\% FBS medium and RPMI1640 plus 5\% FBS medium, respectively, with 32 and $64 \mu \mathrm{g} / \mathrm{mL}$ of TS and incubated at $37^{\circ} \mathrm{C}$ without shaking for $9 \mathrm{~h}$. Hyphal growth was observed under a microscope. $2 \mu \mathrm{g} / \mathrm{mL}$ FLC served as positive control. The bar indicates $20 \mu \mathrm{m}$. 
A

$$
\text { A }
$$

Control

$32 \mu \mathrm{g} / \mathrm{mL}$

$64 \mu \mathrm{g} / \mathrm{mL}$

$128 \mu \mathrm{g} / \mathrm{mL}$

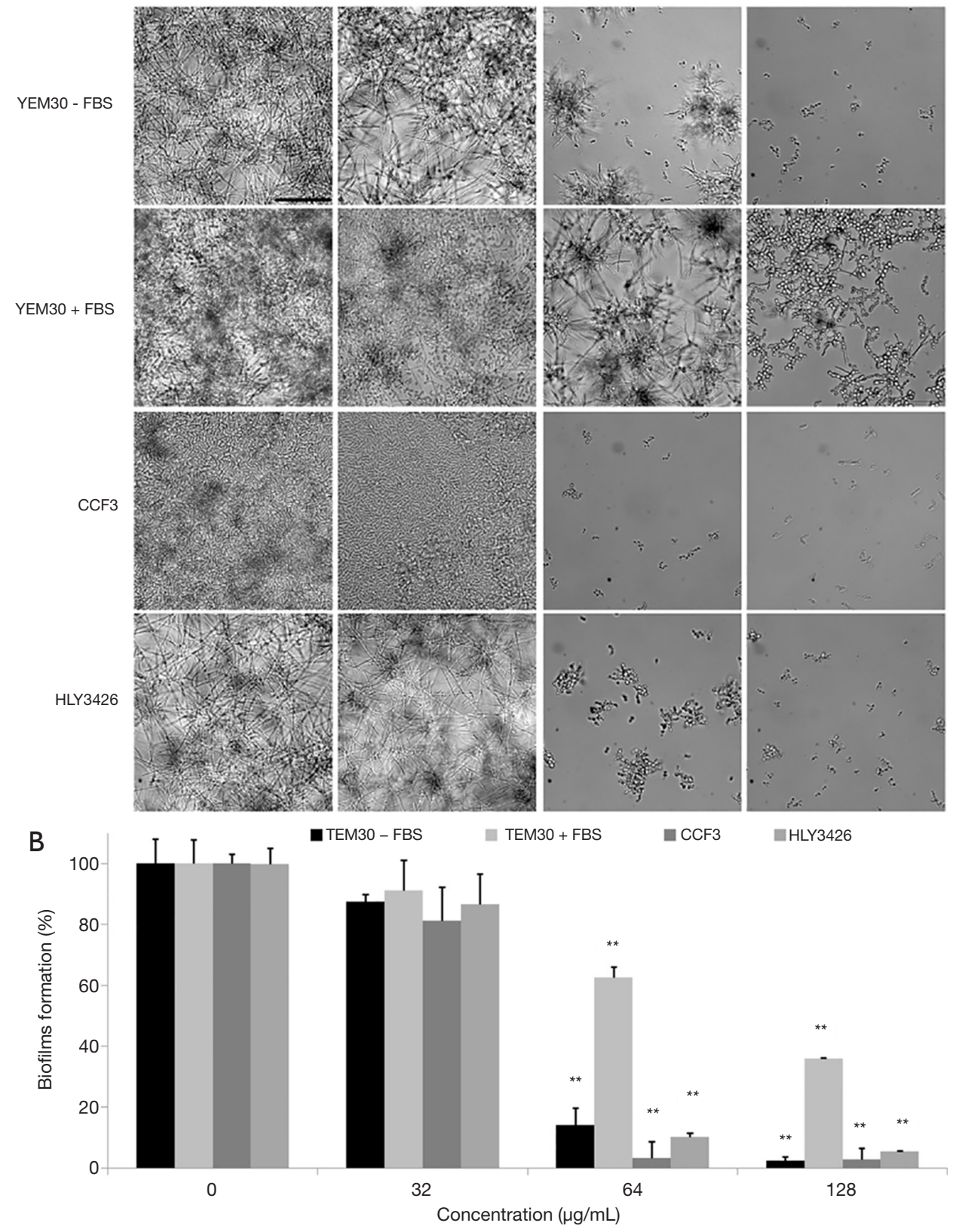

Figure 3 The inhibitory effect of TS against biofilm formation of C. albicans. YEM30, CCF3 and HLY3426 cells were diluted in the RPMI1640 medium or RPMI1640 plus 5\% FBS medium and incubated with different doses of TS at $37^{\circ} \mathrm{C}$ without shaking for $24 \mathrm{~h}$. Microscopic observation (A) and XTT reduction assay (B) were used to detect the formed biofilm in different treatment groups. The bar in (A) indicates $50 \mu \mathrm{m}$. The results in (B) are shown as means \pm standard deviation (SD). Asterisks ${ }^{* *}$ ) represent significance with $\mathrm{P}<0.01$. 


\section{TS inbibits the hyphal and biofilm formation via reducing the level of cAMP content}

Knowing that cAMP occupies the central position in the regulatory network of hyphal development. The decrease of the intracellular cAMP level can weaken the yeast-to-hyphal morphology switch (8), we examined the intracellular cAMP level of $C$. albicans in the presence of TS. As shown in Figure 4A, TS treatment reduced the cAMP level in a dose-dependent manner, and positive control FLC had no significantly effect on the cAMP content. Subsequently, we conducted a cAMP rescue experiment to confirm whether cAMP was involved in TS-induced inhibition. The result showed that $1 \mathrm{mM}$ of exogenous db-cAMP restored the hyphal growth of $C$. albicans in the present of $32 \mu \mathrm{g} / \mathrm{mL}$ TS treatment (Figure $4 B$ ). In addition, exogenous dbcAMP significantly recovered the growth of TS-treated biofilm from $24 \%$ to $48 \%$ (Figure $4 C, \mathrm{P}<0.01$ ) and rebuilt the hyphal and biofilm formation as shown by microscopic observation (Figuire 4D). These results implied that the inhibitory effect of TS against flamentation and biofilm formation was attributed to the decrease of intracellular cAMP content.

\section{TS decreases the transcriptional levels of hypha-specific genes}

Since TS could inhibit the hyphal formation via reducing the cAMP content, the underlying molecular mechanisms were investigated by assessing the transcriptional levels of hypha-specific genes involved in Ras-cAMP-PKA signaling pathway (20). Results showed that TS could significantly downregulate all tested genes (RAS1, ALS3, HWP1, CDC35, EFG1, ECE1) except PDE2, encoding Pde2 protein to hydrolyze intracellular cAMP (Figuire $4 E$ ). These results implied that TS could inhibit flamentation via suppressing the expression of hypha-specific genes.

\section{Repeated exposure does not result in TS-resistance}

Knowing that resistance always complicates the management of fungal infection and limits the use of antifungal drugs, we evaluated the development of TS resistance upon 40-day repeated transfers and exposures to $32 \mu \mathrm{g} / \mathrm{mL}$ (half of MIC) of TS. The results showed that the MIC of TS remained at the level of $64 \mu \mathrm{g} / \mathrm{mL}$ during the 40-day experiment (Figure 5), implying that TS had little potential to cause $C$. albicans to develop resistance after long-time and repeated exposures.

\section{TS shows low cytotoxicity}

Tow normal cell lines and C. elegans were utilized to evaluate the toxicity of TS. The results showed that the $\mathrm{IC}_{50}$ of TS against HT22 (mouse hippocampal cell line) and HEK $293 \mathrm{~T}$ (human embryonic kidney cell line) was 91.17 and $80.69 \mu \mathrm{g} / \mathrm{mL}$, respectively, both of which were lower than the MICs of C. albicans (Table 3). In addition, neither 32 nor $64 \mu \mathrm{g} / \mathrm{mL}$ TS displayed a significantly effect on the survival of worms after 2-day incubation (Figure 6). These results implied that TS had low cytotoxicity in vitro and in vivo.

\section{Discussion}

A long-term therapy is necessary for most fungal infections due to their relapse and obstinacy. However, the adverse effects and drug-resistance usually hinder the management of infection and the usefulness of antifungal agents (24). Meanwhile, they also restrict the clinical application of many antifungal leading compounds. In this study, although TS showed moderate antifungal activity against $C$. albicans, it is worth noting that TS could exert its antifungal action independent of the ATP-binding cassette (ABC) transporters Cdr1 and Cdr2, and the major facilitator Mdr1 as well. Overexpression of efflux pump gene is a vital reason for drug resistance (25), implying that TS should have little chance to form resistance. The following subsequent serial passage experiment showed no significant change of MIC value during the 40-day induction, thus confirming the low resistant potential of TS. What's more, TS, known as a safe compound, indeed displayed low cytotoxicity against normal cell lines and C. elegans. Both low resistance and low toxicity of TS encouraged us to do in-depth research on its further clinical application.

C. albicans is known as the most common opportunistic pathogenic fungus which possesses a number of virulence factors facilitating colonization and infection of the host. The yeast-to-hyphal morphological transition (dimorphism) is considered to represent the most important virulence factor of C. albicans (26). Although both the budding yeast and filamentous hyphal form seem to be important for the pathogenicity of $C$. albicans, hyphal growth is a more critical driver of pathogenesis in that it is more invasive and contributes to host tissue damage (27). In symptomatic mucosal infections, the infected tissues often contain invading hyphae; likewise, hyphal defective mutants of $C$. albicans were found to have low virulence (28). Given the 
A

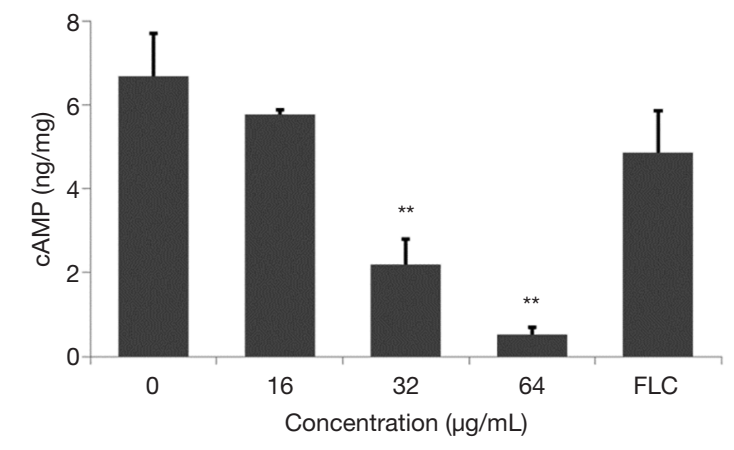

C

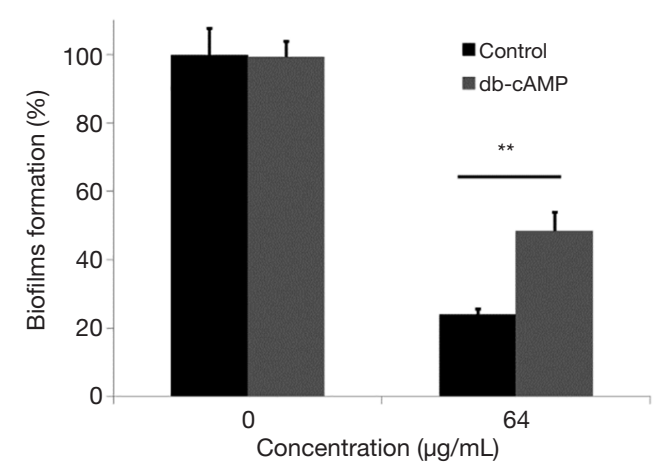

E

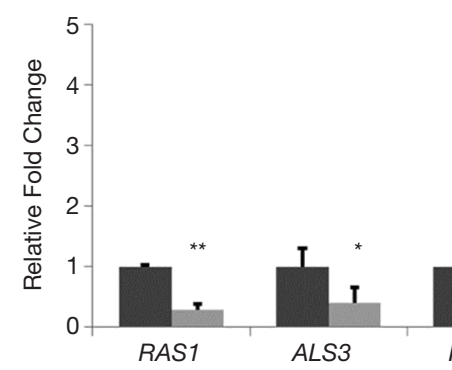

B

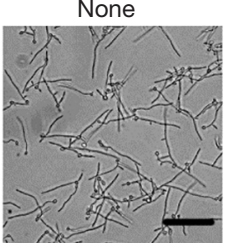

TS
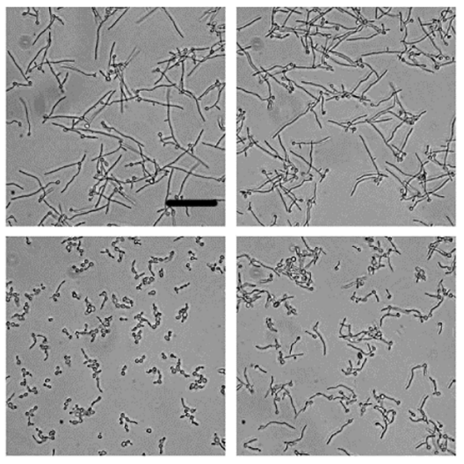

D

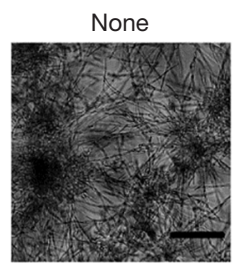

TS
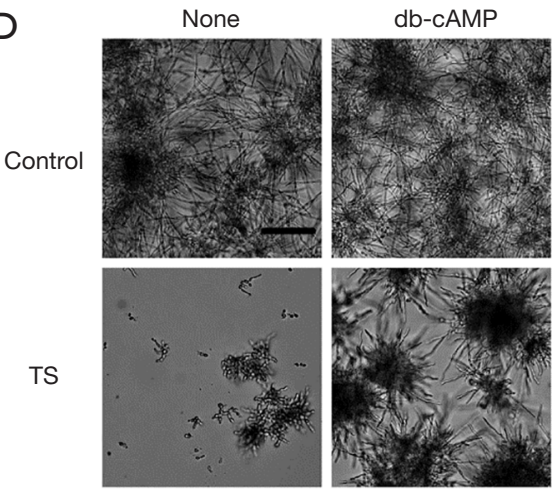

db-cAMP
Control

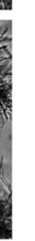




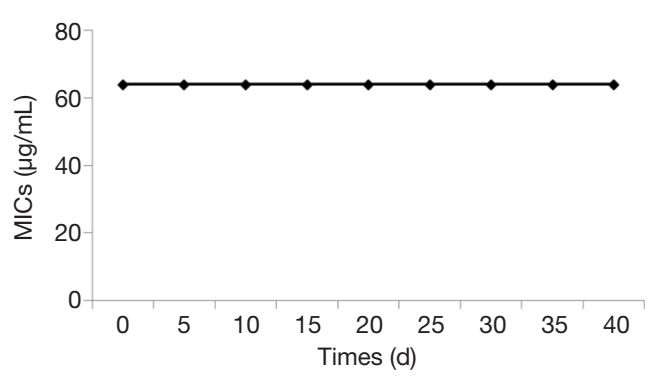

Figure 5 The capacity of TS-resistance formation. Prepared YEM30 cells were diluted in YPD broth and incubated with $32 \mu \mathrm{g} / \mathrm{mL}$ of TS at $30{ }^{\circ} \mathrm{C}, 200 \mathrm{rpm}$. Every day, cells were transferred and measured by serial passage for 40 days. The results are shown as means \pm standard deviation (SD).

Table 3 The $\mathrm{IC}_{50}$ value of TS against different cell lines

\begin{tabular}{lc}
\hline Cell lines & $\mathrm{IC}_{50}(\mu \mathrm{g} / \mathrm{mL})$ \\
\hline HT22 (mouse hippocampal cell line) & 91.17 \\
HEK 293T (human embryonic kidney cell line) & 80.69 \\
\hline
\end{tabular}

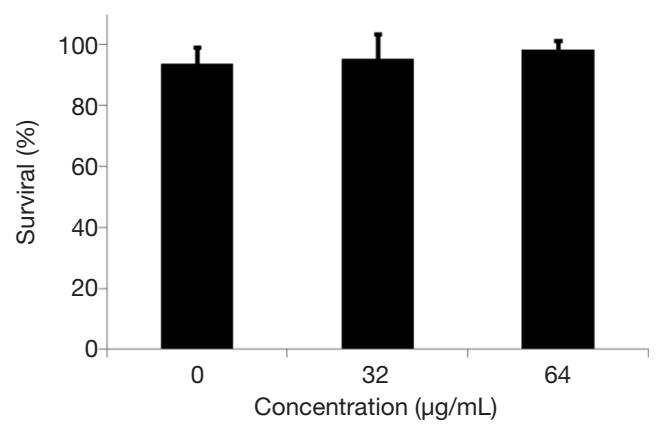

Figure 6 The toxicity of TS against $C$. elegans. Wild type C. elegans $\mathrm{N} 2$ was incubated with different doses of TS in the BHI medium at $25{ }^{\circ} \mathrm{C}$. After 2 -day culture, the worms were observed using a microscope to calculate the survival rates. Results were shown as means \pm standard deviation $(\mathrm{SD})$.

importance of hyphae in virulence, morphological transition of $C$. albicans has been regarded as an important therapeutic target (27).

In the present study, we found that TS could inhibit the yeast-to-hyphal transition in vitro. Since TS treated cells displayed a growth reduction, we used FLC, which is a fungistatic drug affecting growth, also has an effect on hyphae growth because of the growth arrest during the germ tube formation, as a positive control to explored the inhibition of hyphal formation due to the growth reduction or not. Results showed that TS had a similar effect as FLC on the TS treated filamentation, and TS could effectively inhibit the hyphal formation in the hypha-induced serum medium as well. We detected the effect of TS and FLC on the cellular level of cAMP. Results showed that FLC had no significantly effect on the cAMP content, on the contrary, TS could significantly decrease the intracellular cAMP level, which implied that TS acted different modes from FLC to inhibit filamentation and the inhibitory effect was at least not all due to the suppression of proliferation. In addition, in the presence of serum, a strong promoter of filamentation, TS-treated cells could proliferate and remain in the yeast form or develop short hyphae at a low dose, and TS could significantly inhibit biofilm formation at the same concentrations of serum-free medium. These phenomena confirm that this inhibitory effect of TS on filamentation is at least not all due to the suppression of proliferation.

Hyphal growth is triggered by multiple environmental cues and mediated by a series of signal transduction pathways (8). cAMP, known as a second messenger, is crucial for the Ras1-cAMP-Efg1 signaling pathway, which regulates the yeast-to-hyphal transition via controlling the synthesis of cAMP (29). A decrease in intracellular cAMP level could block $C$. albicans in the yeast form irrespective of whether it is under the hypha-induced condition or not (30), implying that a certain level of cAMP is required for filamentation. It was found in our study that TS could decrease the intracellular cAMP level, and exogenous cAMP could rescue the inhibition of hyphae. In addition, the decreased transcriptional levels of hypha-specific genes involved in Ras1-cAMP-Efg1 pathway induced by TS was also obtained. Especially, the downregulation of $C D C 35$ expression, the cAMP synthetic gene, and the upregulation of PDE2, the cAMP hydrolase gene give a molecular reason for the cAMP content reduction. These results suggest that TS could decrease the content of cAMP by affecting the expression of hypha-specific genes to inhibit the yeast-tohyphal transition of $C$. albicans.

Biofilm is structured microbial communities attaching to the surface of biomaterial or non-biomaterial, which consist of mixture of yeast, hyphae and matrix. Hyphal formation often occurs in Candida infections on implanted devices such as indwelling catheters or prosthetic heart valves (31). Crucially, they are seriously resistant to antimicrobial agents due to their limited penetration and high antimicrobial tolerance. Biofilm formation enhances the difficulty of treatment and represents one of the most 
well-known virulence factors. Our work showed that TS could significantly retard biofilm formation. To detect the inhibitory effect via inhibition of filamentation or proliferation, the non-filamentous and its complementation strains were used to test the effect of TS on their biofilm formation. As TS has a similar inhibitory effect on the two strains, the suppression of biofilm formation induced by TS is partial to the suppression of proliferation. Moreover, exogenous cAMP could rescue the inhibition of biofilm formation induced by TS as well, implying that both the inhibition of proliferation and filamentation result in the suppression of biofilm formation.

In summary, we evaluated the cytotoxicity and antivirulence activity of TS against $C$. albicans, and preliminarily explored its action mode of hyphal inhibition. Our work may provide a friendly antifungal natural product to expand the current antifungal agent arsenal via blocking the virulence factors of $C$. albicans.

\section{Acknowledgments}

Funding: This research was supported by National Natural Science Foundation of China (81701390, 81871734, 81902040), Natural Science Foundation of Jiangsu Province (BK20170250), Postdoctoral Foundation of Jiangsu Province (2018K245C), Jiangsu Provincial Medical Talent (ZDRCA2016053), Six Talent Peaks Project of Jiangsu Province (WSN-135), Advanced Health Talent of SixOne Project of Jiangsu Province (LGY2016042), Xuzhou Science and Technology Innovation Project (KC19057).

\section{Footnote}

Conflicts of Interest: GB serves as an unpaid managing editor of Annals of Translational Medicine. The other authors have no conflicts of interest to declare.

Ethical Statement: The authors are accountable for all aspects of the work in ensuring that questions related to the accuracy or integrity of any part of the work are appropriately investigated and resolved. The study was approved by the ethics committee at Affiliated Hospital of Xuzhou Medical University, the number of the approval was XXYFY2019-KL141-01.

Open Access Statement: This is an Open Access article distributed in accordance with the Creative Commons Attribution-NonCommercial-NoDerivs 4.0 International
License (CC BY-NC-ND 4.0), which permits the noncommercial replication and distribution of the article with the strict proviso that no changes or edits are made and the original work is properly cited (including links to both the formal publication through the relevant DOI and the license). See: https://creativecommons.org/licenses/by-nc-nd/4.0/.

\section{References}

1. Kato H, Yoshimura Y, Suido Y, et al. Prevalence of, and risk factors for, hematogenous fungal endophthalmitis in patients with Candida bloodstream infection. Infection 2018;46:635-40.

2. Pfaller MA, Diekema DJ. Epidemiology of invasive mycoses in North America. Crit Rev Microbiol 2010;36:1-53.

3. Sobel JD. Vulvovaginal candidosis. Lancet 2007;369:1961-71.

4. Foxman B, Marsh JV, Gillespie B, et al. Frequency and response to vaginal Symptoms among white and African American women: Results of a Random Digit Dialing Survey. J Womens Health 1998;7:1167-74.

5. Bongomin F, Gago S, Oladele RO, et al. Global and multinational prevalence of fungal diseases-estimate precision. J Fungi (Basel) 2017;3:57.

6. Mayer FL, Wilson D, Hube B. Candida albicans pathogenicity mechanisms. Virulence 2013;4:119-28.

7. Finkel JS, Mitchell AP. Genetic control of Candida albicans biofilm development. Nat Rev Microbiol 2011;9:109-18.

8. Sudbery PE. Growth of Candida albicans hyphae. Nat Rev Microbiol 2011;9:737-48.

9. Höfs S, Mogavero S, Hube B. Interaction of Candida albicans with host cells: virulence factors, host defense, escape strategies, and the microbiota. J Microbiol 2016;54:149-69.

10. Pierce CG, Chaturvedi AK, Lazzell AL, et al. A novel small molecule inhibitor of Candida albicans biofilm formation, filamentation and virulence with low potential for the development of resistance. NPJ Biofilms Microbiomes 2015;1:15012.

11. Newman DJ, Cragg GM. Natural Products as Sources of New Drugs from 1981 to 2014. J Nat Prod 2016;79:629-61.

12. Xia C, Zhu Q, Tian J, et al. Surface activity of tea saponin and the related functional properties. Journal of Tea Science 1990;10:1-10.

13. Hao W, Zhong G, Hu M, et al. Control of citrus postharvest green and blue mold and sour rot by 
tea saponin combined with imazalil and prochloraz. Postharvest Biology \& Technology 2010;56:39-43.

14. Sanglard D, Ischer F, Monod M, et al. Susceptibilities of Candida albicans multidrug transporter mutants to various antifungal agents and other metabolic inhibitors. Antimicrob Agents Chemother 1996;40:2300-5.

15. Sun L, Sun S, Cheng A, et al. In vitro activities of retigeric acid $\mathrm{B}$ alone and in combination with azole antifungal agents against Candida albicans. Antimicrob Agents Chemother 2009;53:1586-91.

16. Cao F, Lane S, Raniga PP, et al. The Flo8 transcription factor is essential for hyphal development and virulence in Candida albicans. Mol Biol Cell 2006;17:295-307.

17. Li Y, Chang W, Zhang M, et al. Synergistic and drugresistant reversing effects of diorcinol D combined with fluconazole against Candida albicans. FEMS Yeast Res 2015; 15 :fov001.

18. CLSI. Reference Methods for Broth Dilution Antifungal Susceptibility Testing of Yeast; Approved Standard, CLSI Document M27-A3, 3rd ed. Wayne, PA: Clinical and Laboratory Standards Institute, 2008.

19. Li Y, Shan M, Yan M, et al. The inhibition of transcinnamaldehyde on the virulence of Candida albicans via enhancing farnesol secretion with low potential for the development of resistance. Biochem Biophys Res Commun 2019;515:544-50.

20. Li Y, Chang W, Zhang M, et al. Natural product solasodine-3-O- $\beta$-D- glucopyranoside inhibits the virulence factors of Candida albicans. Fems Yeast Research 2015; 15 :fov060.

21. Jung WH, Stateva LI. The cAMP phosphodiesterase encoded by CaPDE2 is required for hyphal development in Candida albicans. Microbiology 2003;149:2961-76.

Cite this article as: Li Y, Shan M, Li S, Wang Y, Yang H, Chen Y, Gu B, Zhu Z. Teasaponin suppresses Candida albicans filamentation by reducing the level of intracellular cAMP. Ann Transl Med 2020;8(5):175. doi: 10.21037/atm.2020.01.124
22. Li Y, Chang W, Zhang M, et al. Diorcinol D exerts fungicidal action against Candida albicans through cytoplasm membrane destruction and ROS accumulation. PLoS One 2015;10:e128693.

23. Douglas LJ. Candida biofilms and their role in infection. Trends in Microbiology 2003;11:30-6.

24. Yu Y, Li J, Wang S, et al. Effect of Candida albicans bronchial colonization on hospital-acquired bacterial pneumonia in patients with systemic lupus erythematosus. Ann Transl Med 2019;7:673.

25. Cowen LE, Dominique S, Howard SJ, et al. Mechanisms of antifungal drug resistance. Cold Spring Harb Perspect Med 2014;5:a019752.

26. Biswas S, Van Dijck P, Datta A. Environmental sensing and signal transduction pathways regulating morphopathogenic determinants of Candida albicans. Microbiol Mol Biol Rev 2007;71:348-76.

27. Jacobsen ID, Wilson D, Wächtler B, et al. Candida albicans dimorphism as a therapeutic target. Expert Rev Anti Infect Ther 2012;10:85-93.

28. Jabra-Rizk MA, Kong EF, Tsui C, et al. Candida albicans pathogenesis: fitting within the host-microbe damage response framework. Infect Immun 2016;84:2724-39.

29. Lindsay AK, Deveau A, Piispanen AE, et al. Farnesol and cyclic AMP signaling effects on the hypha-toyeast transition in Candida albicans. Eukaryot Cell 2012;11:1219-25.

30. Zhang M, Chang W, Shi H, et al. Biatriosporin D displays anti-virulence activity through decreasing the intracellular cAMP levels. Toxicol Appl Pharmacol 2017;322:104-12.

31. Hall CW, Mah TF. Molecular mechanisms of biofilmbased antibiotic resistance and tolerance in pathogenic bacteria. FEMS Microbiol Rev 2017;41:276-301. 